\title{
Gruppenmoderation - Neuere Entwicklungen und Überlegungen
}

\author{
Erich H. Witte
}

Die Bedeutung kleiner Gruppen für das menschliche Dasein kann kaum überschätzt werden. Unsere soziale Praxis nimmt diese Bedeutsamkeit auf und bildet aktiv kleine Gruppen, wenn es schwieriger wird: Einzelrichter vs. Schöffengericht, Minister vs. Kabinett, Doktorvater vs. Promotionsprüfungsausschuss etc. Implizit glauben wir, dass bereits durch die Bildung dieser Gruppen, Gremien, Ausschüsse die Qualität der vorgeschlagenen Lösung auf ein höheres Niveau gehoben wird. Dass es sich hierbei vielfach um eine Illusion handelt, kann man an Planungsfehlern (Elbphilharmonie, Berliner Flugplatz, Stuttgart 21,...) oder auch Entscheidungen mit katastrophalem Ausgang wie z. B. in der bemannten Raumfahrt (Challenger), militärischer Interventionen oder Finanzspekulationen(Lehman Brothers oder deutsche Landesbanken) entnehmen. Sicherlich sind Fehlentscheidungen auch durch optimal angeleitete Gruppen nicht generell vermeidbar, aber wir haben uns bisher noch nicht darauf eingestellt, die Qualität von Gruppenlösungen zu hinterfragen und zu optimieren. Gruppen können uns in der Tat helfen, unsere Lebenswelt besser zu bewältigen, dazu jedoch muss man genauer hinschauen, unter welchen Bedingungen und mit welcher Vorgehensweise diese Gruppenentscheidungen höherer Qualität erreichbar sind. Sie stellen sich nämlich nicht automatisch ein. Bisher haben wir uns noch zu wenig darüber Gedanken gemacht, wie man dieses unterstellte Ziel der optimalen Lösungsqualität durch Gruppen auch erreichen kann. Viele alltagspsychologische Ideen sind Grundlage für das gemeinsame Vorgehen und Scheitern in der Gruppeninteraktion (Harmonie, Kohäsion, Konfliktvermeidung, Gleichbehandlung), teilweise werden diese Ziele auch von außen durch professionelle Moderatoren verfolgt. Fast immer fehlen dabei die theoretische Fundierung und die empirische Überprüfung, denn die konkrete Aufgabenstellung muss jeweils das spezifische Vorgehen bestimmen und nicht etwa als generelles Prinzip die Harmonie in der Gruppe (Witte 2012). Wenn diese Voraussetzungen aber geschaffen wurden, empirisch gut gestützte Moderationsmethoden für die spezifischen Problemstellungen existieren und gut ausgebildete ModeratorInnen eingesetzt werden können, dann hat man, ähnlich wie bei der Entwicklung von

Online publiziert: 06.05 .2014

(C) Springer Fachmedien Wiesbaden 2014

Prof. Dr. E. H. Witte $(\bowtie)$

Fakultät für Psychologie und Bewegungswissenschaft, Universität Hamburg,

Hamburg, Deutschaland

E-Mail: erichwitte@aol.com 
neuen PKWs, Assistenzsysteme zur Verfügung, die im übertragenen Sinne Verkehrstote verringern, Unfälle vermeiden und einen wohl angepassten, sparsamen Verkehrsfluss ermöglichen. Sich z. B. der Einparkhilfe zu überlassen, trifft zuerst einmal auf Skepsis. Erst über die Erfahrung mit diesen Assistenzsystemen wird man diese schätzen lernen. Ähnlich ist es mit der Assistenz durch GruppenmoderatorInnen, die muss man auch erst schätzen gelernt haben, bevor man auf sie vertraut. In Zukunft werden auch elektronische Hilfsmittel für ihre Durchführung eingesetzt werden (Unger und Witte 2007). Bisher hat man noch allzu sehr auf die menschlichen Fähigkeiten vertraut und bei der Moderation auf die menschlichen Anlagen durch die Evolution gesetzt (siehe Witte, in diesem Bd.). In gewissen Bereichen ist das auch erfolgreich, aber führt in vielen Bereichen auch zu katastrophalen Ergebnissen (Seelheim und Witte, in diesem Heft). Es macht eben wenig Sinn mit einem Smart im Gelände zu fahren oder mit einem Landrover (SUV) in der Innenstadt parken zu wollen. Auch ein Formel-1-Rennen wird man nur mit einer Spezialkonstruktion gewinnen können. Wahrscheinlich befinden wir uns beim Umgang mit Gruppen noch auf dem Niveau der anfänglichen Motorisierung. Wenn man das Potenzial dieser kleinen Gruppen erkannt haben wird, dann werden auch Forschung und Entwicklung verstärkt einsetzen, um die Probleme angemessen anzugehen. Dieses Heft kann ein Funke sein, der in der Praxis die Nachfrage auslöst, sich bei den entsprechenden Problemstellungen eine professionelle Moderation einzukaufen, weil man nicht scheitern darf oder das beste Ergebnis erzielen möchte (Fischer, in diesem Heft). Man muss sich aber aktiv in diesem Bereich umschauen, damit man immer die neueste Entwicklung mitbekommt. Auf dem technischen Gebiet ist das für viele Akteure eine Selbstverständlichkeit, auf dem sozialen Gebiet vertrauen wir eher der Küchenpsychologie oder implizit der Evolution und sozialen Repräsentation. Dann wird auch deutlich, dass die Aufgaben eines Vorgesetzten und eines Gruppenmoderators sehr verschieden sind, zumindest wenn man sich moderne Entwicklungen anschaut (Graf, in diesem Heft). Ein guter Vorgesetzter ist nicht zwingend ein guter Moderator oder sollte überhaupt diese Rolle einnehmen.

Als unausgesprochene Voraussetzung handelt es sich bei diesen Themen um die Kombination individueller Positionen zu einem gemeinsamen Ergebnis. Das gemeinsame Ziel ist bei allen Gruppenmitgliedern die Voraussetzung. Nun gibt es aber auch Situationen, in denen diese Gemeinsamkeit überhaupt erst hergestellt werden muss, um als Gruppe zu agieren. Dieses kann man methodisch herbeiführen, indem man einen Reflexionsprozess in Gang setzt, der über die Herstellung einer Box mit einer Außen- und einer Innenperspektive vorgenommen wird (Tacke und Knitter, in diesem Heft). Die Gruppenaufgabe besteht dann darin, diese Box in der Gruppensituation zu präsentieren, wobei es offen bleibt, wer welche Inhalte im Innern der Box zu sehen bekommt. Die Aufgabe ist die Herstellung einer Gruppensituation von Personen, die sich nicht kannten, aber in Zukunft gemeinsam eine Wohn-, Lehr- und Lernumwelt teilen sollen. Eine solche Methode sollte nicht einfach auf andere Aufgabenstellungen übertragen werden (Projektgruppen), weil diese Aufgaben sich erheblich unterscheiden. Gruppenmoderationstechniken sind prinzipiell aufgabenbezogen zu entwickeln und einzusetzen. Teilweise eben auch in Abhängigkeit von den Eigenschaften und Vorstellungen der Teilnehmer.

In einem weiteren Artikel (Redlich und Rogmann, in diesem Heft) sind die unterschiedlichen Positionen bereits manifest, weil es sich um eine Konfliktmoderation mit mehreren Parteien handelt. Eine solche Ausgangsbedingung gehört sicherlich zu den 
schwierigsten Verhandlungen, die es gibt. Hier eine gemeinsame Lösung zu erarbeiten, ist ohne Moderation kaum denkbar, aber auch mit Hilfe einer Moderation sehr schwierig. Die Forschung auf diesem komplexen Gebiet der Mehr-Parteien- Konflikt-Moderation ist kaum vorhanden. Selbst auf dem Gebiet der Zwei-Parteien-Mediation ist der Forschungsstand nicht sehr angewachsen (vergl. Witte 1994). Hier müssten Forschung, Theorienbildung und Methodenentwicklung verstärkt einsetzen, weil viele Konflikte dieser Art gefährlich sind und höchst aggressive Handlungen zur Folge haben können.

Im letzten Beitrag geht es dagegen um die Herstellung von (kognitiver) Abweichung, nämlich um die Entwicklung origineller Ideen, die eben nicht bereits allen bekannt sind (Krieger und Witte, in diesem Heft). Neben der nicht mehr wegzudenkenden Methode des Gruppen-Brainstorming, die viel Spaß macht, ein gutes Klima erzeugt und nur mittelmäßige Ideen produziert, hat sich das Brainwriting etabliert. Das macht weniger Spaß, ist aber häufig qualitativ besser. Der empirischen Forschung stellt sich dann die Frage, ob es aus theoretischen Gründen eine Verbesserung dieser Methode gibt. Sie muss natürlich empirisch überprüft werden. Erste Hinweise auf diese Verbesserungsmöglichkeit ergeben sich durch frühe Erkenntnisse über das Ausschöpfen eines Assoziationsreservoirs, wobei bekannte Ideen zuerst ins Gedächtnis kommen. Lässt man nun gezielt diese bekannten Ideen zuerst produzieren, dann kann man hoffen, in einer zweiten Phase eher originelle Ideen zu erhalten. Dieses Ergebnis kann man im Ansatz finden. Vielleicht kann man in Zukunft dadurch gezielter kreative Ideen erhalten.

Was sind die Aufgaben dieses Heftes?

1. Die große Bedeutung von aufgabenbezogener Gruppenmoderation zu verdeutlichen.

2. Gruppenmoderation und andere Formen der Gruppenleitung zu unterscheiden.

3. Die Hindernisse in der Anwendung von Gruppenmoderation zu benennen.

4. Auf neue Methoden der persönlichen Gruppenintegration und der kognitiven Abweichung aufmerksam zu machen.

5. Die Forschungsdefizite bei der Mehr-Parteien-Konflikt-Moderation zu benennen.

Letztlich sollten die Verantwortlichen bei relevanten Gruppenentscheidungen ein schlechtes Gewissen bekommen, wenn sie keine professionelle Gruppenmoderation eingesetzt haben und ein Ergebnis nicht den Ansprüchen genügt. Ferner sollten die Entscheidungsträger das Potenzial einer aufgabenzentrierten Gruppenmoderation für ihren Bereich erkennen. Schließlich sollte sich Forschung diesem Gebiet verstärkt zuwenden, um immer bessere und differenziertere Methoden zu entwickeln, die dann in einer Ausbildung zur Verfügung gestellt werden können.

\section{Literatur}

Unger, D., \& Witte, E. H. (2007). Virtuelle Teams - Geringe Kosten, geringer Nutzen? Zur Leistungsverbesserung von Kleingruppen beim Problemlösen durch elektronische Moderation. Gruppendynamik und Organisationsberatung, 38, 165-184.

Witte, E. H. (Hrsg.). (1994). Mediation. Gruppendynamik, 25, 237-318.

Witte, E. H. (2012). Gruppen aufgabenzentriert moderieren. Theorie und Praxis. Göttingen: Hogrefe. 